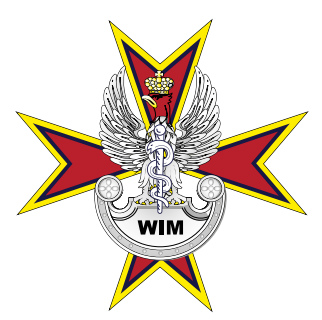

\title{
MUCORMYCOSIS AND COVID-19
}

\author{
Mukormykoza i COVID-19
}

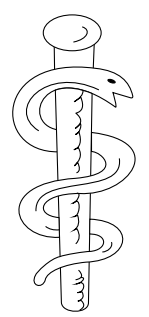

\section{Jacek Zbigniew Kubiak ${ }^{1,2}$}

1. Department of Experimental Embryology, Institute of Genetics and Animal Biotechnology of the Polish Academy of Science, Polska

2. Cell Cycle Group, Faculty of Medicine, Univ Rennes, UMR 6290, CNRS, Institute of Genetics and Development of Rennes (IGDR), France

\begin{abstract}
This article presents the current situation regarding the spread of mucormycosis. Since mucormycosis is a very poorly known disease in Poland, we present here only the most important facts and observations regarding the relationship of this mycosis with COVID-19. We are dealing with a wave of infections, mainly in India, caused by the so-called black fungus. The increase in the number of infections and their spread also outside of India is directly related to the COVID-19 pandemic, and more specifically to the use of drugs containing corticosteroids or anti-interleukins, such as tocilizumab, which suppress the patients' immune system. There are voices of specialists warning against underestimating mucormycosis and related mycoses, such as aspergillosis, and calling for more research on these diseases to find effective treatments and eventual preventive measures.
\end{abstract}

Keywords: mucormycosis, COVID-19, immunodepression, mycoses, aspergillosis.

DOI $10.53301 / / \mathrm{w} / 145908$

Received: $2021-10-12$

Accepted: 2022-01-17

\section{Rapid increase in the number of cases in India in 2021}

Mucormycosis, which is a severe and often fatal invasive fungal infection, achieved wider public awareness during the COVID-19 outbreak in India in early 2021 [1,2]. The rapid increase in mucormycosis cases followed the second wave of the COVID-19 pandemic in India caused by the SARS-CoV-2 delta variant. This increase was noticed especially in the COVID-19 survivors and in diabetic patients. Mucormycosis is caused by fungi of the order Mucorales. The most common species is Rhizopus oryzae, but in tropical countries, there are many different species, belonging to the genera Rhizopus, Apophysomyces, Mucor, and Lichtheimia, which can also cause mucormycosis [3]. However, the precise identification of the species in the patients is very difficult. Spores and mycelial fragments are found in the soil, and therefore also in the ubiquitous dust scattered everywhere in the air. Mucormycosis is not a new disease to India, but before COVID-19 pandemic it occurred sporadically in the immunocompromised people. The COVID-19 pandemic has caused the number of such patients to skyrocket. For this reason the mucormycosis, next to COVID-19, has become the second epidemic in this country [4].

Mucormycosis is extremelly fatal disease with $50 \%$ mortality rate. The fungus enters the human body through the mucosa during breathing. That is why it attacks the nasal sinuses so often. But it can also pass through the

\section{Corresponding author}

Jacek Z. Kubiak

UMR 6290, CNRS/UR1, IGDR, Faculty of Medicine;

2 Ave. du Prof. Leon Bernard, 35043 Rennes cedex,

France

Phone: 00-33-223234698

e-mail: jacek.kubiak@univ-rennes1.fr

skin wounds, or the circulatory system to the rest of the body. Because it's ubiquitous, the fungus easily attacks the COVID-19 patients with weakened immune system.

Additional risk factors for mucormycosis are: ketoacidosis occurring in cases of poorly treated or untreated diabetes (which is also an increased risk factor for COVID-19) as the fungus grows particularly well in the acidified tissues, patients dialyzed and undergoing deferoxamine therapy for kidneys diseases, patients with extensive wounds or burns, or in case of malnutrition that is very common in overpopulated India.

India has been struggling with the COVID-19 pandemic during 2021. The SARS-CoV-2 virus delta variant has proved to be particularly deadly in this densely populated country that often lacks high-quality healthcare. The situation became even more complicated with the emergence of new mutations of the delta variant, called the delta plus. But the mucormycosis accompanying the COVID-19 pandemic has proven to be a particularly gruesome scourge. The SARS-CoV-2 infection itself greatly weakens the body and immune system. In addition, treatments of patients suffering from COVID-19 with drugs containing corticosteroids or anti-interleukins reduces the activity of the patients' immune systems to such an extent that their state resembles the immunosuppression applied, for example in the transplant recipients to prevent acute transplant rejection [5]. This is how the pa- 
tients recovering from COVID-19 infection, often suffer from mukormycosis.

Mucormycosis manifests with the black color of the tissues affected by mycosis - hence the common name of the disease - the black fungus. The disease is usually localized in the tissues of the sinuses, the eye, lungs, or the skin around the wound6. But it can also take the form of spread infection in the body called the disseminated form. This happens when the fungus travels in the bloodstream through the body. In the case of eye or paranasal sinus infections, mucormycosis can easily spread to the brain, which is always associated with a fatal prognosis for the patient. Therefore, in such cases, the eye or sinus areas sare surgically removed. Obviously, such a drastic medical intervention causes enormous injuries to the face and head. This is why local people fear mucormycosis so much - it is not only deadly but also mutilates people who managed to survive COVID-19.

\section{Spreading beyond India}

To date, the vast majority of reported cases of mucormycosis in the world have occurred in India. The incidence of mucormycosis is 70 times higher in India than in the other parts of the world [3]. So, it is not surprising that the particularly rapid increase in the number of infections in connection with the COVID-19 pandemic happened in India. However, the spread of mucormycosis to India's neighboring countries is very worrying. Many cases are reported not only in neighboring Pakistan but also in the countries east and north of India such as $\mathrm{Ne}$ pal, China, Bangladesh, Malaysia, and even Indonesia [7]. In the latter, mainly in Java and Bali, the particularly high level of infections with the delta variant SARS-CoV-2 was recorded, and consequently, the greater number of cases of mucormycosis followed. The COVID-19 pandemic increased the incidence of mukormycosis also in Soth America, mostly Brasil $[8,9])$.

This new epidemic is of great concern to the Indonesian authorities. The health authorities started a campaign to propagate knowledge about mucormycosis and its prevention [10]. Of course, an early diagnosis of mucormycosis gives better chances for its treatment. For this reason, the media report the typical symptoms of this disease, which, unfortunately, are not very specific at the beginning of the disease. These include fever with a cough, chest pain, and shortness of breath that do not improve with standard treatments. In cases of infection through the intestinal mucosa, abdominal pain, nausea, vomiting, and gastrointestinal bleeding occur. Particular attention is also paid to the infections of skin wounds, e.g. after surgery or burns, where black blisters or ulcers occur. The population is warned of disseminated forms of mucormycosis, which can spread to internal organs, including the brain, spleen, heart, and which manifests itself as a severe disease with specific symptoms difficult to recognize. The disease can also cause coma and changes in mental status.

Due to global warming, mucormycosis can also reach our latitudes. Fungi causing mucormycosis can also be brought to the western coutnries along with the dust containing spores or mycelial fragments. The first reports of mucormycosis also appear in Poland, especially in patients with poorly treated diabetes. However, for now, aspergillosis is probably the greater threat in Poland. It is mucormycosis-like mycosis caused by the aspergillus, a fungus of the genus Apergillus, and Candida sp. Aspergillosis and candidosis also occur most frequently in immunocompromised individuals, such as AIDS patients, and also highly increased as the consequence of COVID-19 pandemic [11]. These patients are either treated with amphotericin B, itraconazole, or voriconazole, or surgical treatment is also used if necessary.

\section{Urgent need for more research on mucormyosis and related mycoses}

In the August 2021 issue of Lancet Microbe, published online in June 2021, three researchers Neil Stone, Nitin Gupta, and Ilan Schwartz of the Hospital for Tropical Diseases, University College London, Kasturba Medical College in Manipal, India, and the University of Alberta in Edmonton, Canada, call for increased efforts to improve diagnosis and treatment of mycoses such as mucormycosis and aspergillosis [12]. They argue that these diseases, which until now were considered to be secondary because they were restricted to a small group of particularly susceptible patients, now can threaten anyone. The COVID-19 pandemic should also change the approach to this type of forgotten but extremely dangerous disease.

They point out that the most often used antifungal treatment is amphotericin B, a nephrotoxic polyene antifungal, which is in use since 1958. Liposomal formulations, preferred because of reduced toxicity, are often too expensive or even unavailable in many poor countries. Few alternative medications, such as posaconazole and isavuconazole, are even more expensive and therefore out of reach for poor populations.

The mucormycosis epidemic in India, but also in Indonesia and Brasil, has made it clear how huge is the problem of fungal infections and how poor is the state of medical research necessary for correect prevention, diagnosis and efficient and cheap treatments available for for countries threatened by this scourge.

This major global warning should stimulate action to tackle the many problems associated with this disease. There is a need to better understand the risk factors that contribute to the current epidemic. It is very important to develop fast, reliable and non-invasive or minimally invasive diagnostics of mucormycosis, to increase access to existing treatments and to improve therapeutic measures.

\section{Literature}

1. World Health Organization (WHO). The current COVID-19 situation. India. Accessed on June 15 2021: https://www. who.int/countries/ind/

2. Jeong W, Keighley C, Wolfe R, Lee WL, Slavin MA, Kong $D C M$, et al. The epidemiology and clinical manifestations of mucormycosis: a systematic review and meta-analysis of case reports. Clin Microbiol Infect. 2019; 25 (1): 26-34.

3. Prakash $\mathrm{H}, \mathrm{Chakrabarti} \mathrm{A}$. Epidemiology of mucormycosis in India. Microorganisms. 2021; 9: 523 
4. Biswas S. Mucormycosis: the 'black fungus' maiming Covid patients in India. https://www.bbc.co.uk/news/world-asia-india-57027829; May 9, 2021.

5. Moorthy A, Gaikwad R, Krishna S, Hegde R, Tripathi KK, Kale PG, et al. SARS-CoV-2, uncontrolled diabetes and corticosteroids-an unholy trinity in invasive fungal infections of the maxillofacial region? A retrospective, multi-centric analysis. J Maxillofac Oral Surg. 2021: 1-8.

6. Misra SR, Das R, Mohanty N. Sinomaxillary mucormycosis an increasingly common occurrence in the COVID-19 pandemic. BMJ Case Rep. 2021 Sep 6; 14 (9): e244811. doi: 10.1136/bcr-2021-244811

7. Song G, Liang G, Liu W. Fungal Co-infections Associated with Global COVID-19 pandemic: a clinical and diagnostic perspective from China. Mycopathologia. 2020; 185 (4): 599-606.

8. Farias LABG, Damasceno LS, Bandeira SP, Barreto FKA, Leitão TDMJS, Cavalcanti LPG. COVID-19 associated Mucormycosis (CAM): Should Brazil be on alert? Rev
Soc Bras Med Trop. 2021 Sep 6; 54:e 04102021. doi: 10.1590/0037-8682-0410-2021. eCollection 2021.

9. Nucci M, Engelhardt M, Hamed K. Mucormycosis in South America: A review of 143 reported cases. Mycoses. 2019; 62 (9): 730-8.

10. Kubiak J. Czarny grzyb atakuje chorych na COVID-19 poza Indiami. Mukormykoza dotarła do Pakistanu i Indonezji. https://wyborcza.pl/TylkoZdrowie/7,137474,27469307, czarny-grzyb-atakuje-chorych-na-covid-19-poza-indiami-mukormykoza.html Gazeta Wyborcza

11. Santana MF, Pivoto G, Alexandre MAA, Baía-da-Silva DC, Borba MGDS, Val FA, et al. Confirmed Invasive Pulmonary Aspergillosis and COVID-19: the value of postmortem findings to support antemortem management. Rev Soc Bras Med Trop. 2020; 53: e20200401.).

12. Stone N, Gupta N, Schwartz I. Mucormycosis: time to address this deadly fungal infection, The Lancet Microbe 2(8)e343-e344, August 01, 202, DOI: https://doi. org/10.1016/S2666-5247(21)00148-8).

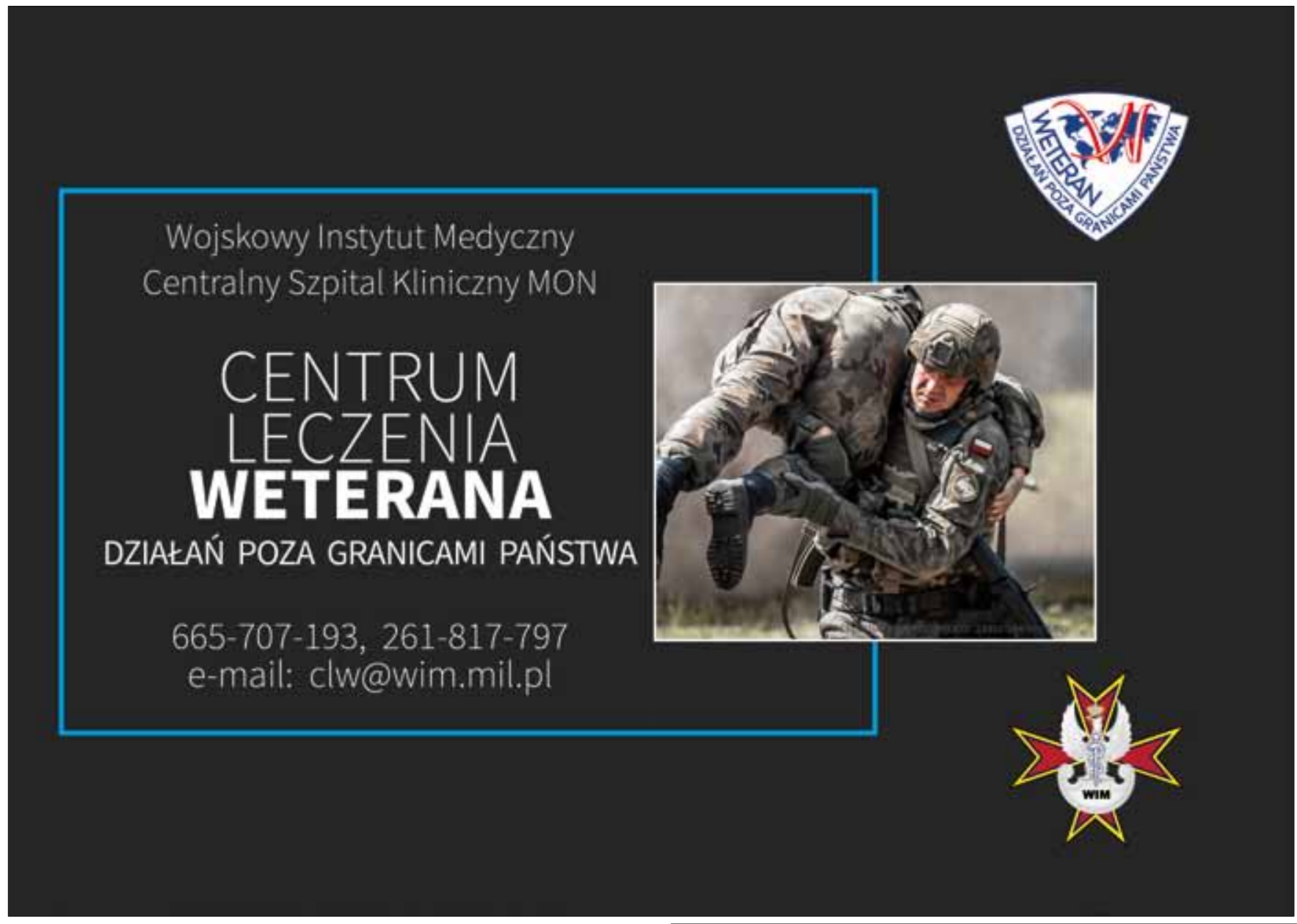

